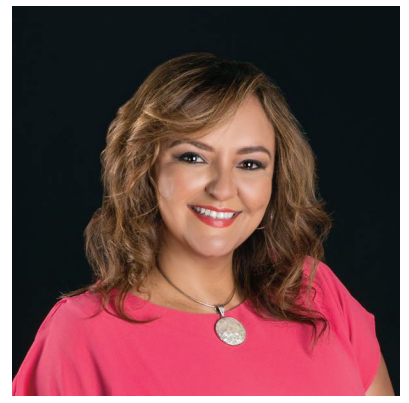

Dra. Jenny Guerrero

Ecuador

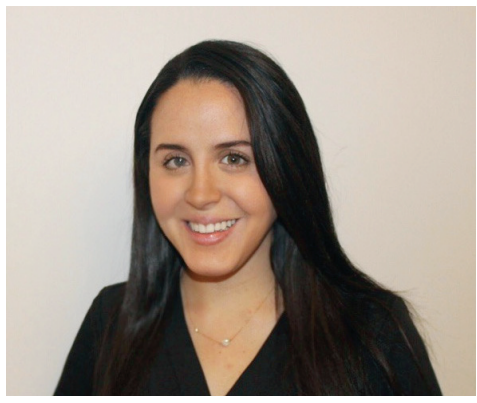

Od. Camila Peralta

Ecuador

\title{
Análisis tomográfico de variaciones anatómicas de premolares en la clínica nexodent, Guayaquil 2016.
}

\author{
Dra. Jenny Guerrero Ferreccio ${ }^{1}$ \\ Od. Camila Adriana Peralta Mier ${ }^{2}$
}

Autor de correspondencia: endofile@hotmail.com

\section{Resumen}

Introducción: El tratamiento endodontico representa en la actualidad una gran rama en el área de la odontología de importancia para la preservación de piezas dentales, que se verían afectadas por el ataque de agentes externos y que comprometen su funcionabilidad y estética, pero la complejidad de la anatomía de los conductos de todos los dientes en general aún sigue siendo un problema, más aun en los premolares, ya que estos a diferencia de las demás piezas dentarias, tienen diferentes formas y cantidad de conductos radiculares. ${ }^{(6)}$

Propósito: El propósito de este estudio es demostrar la importancia del conocimiento sobre la anatomía radicular y sus variaciones anatómicas, para minimizar el fracaso al realizar una terapia endodontica.

Especialista en Endodoncia UAG, México.

Correspondencia: Jenny Guerrero Ferreccio e-mail: endofile@hotmail.com

2 Odontóloga UCSG, Ecuador.

\section{Tomographic analysis of anatomical variations of premolars in the clinic Nexodent, Guayaquil 2016}

Fecha de recepción: Julio 2018

Fecha de aceptación: Septiembre 2018

Objetivo: El objetivo directo de este estudio es determinar las variaciones anatómicas de premolares superiores e inferiores y su relación con estructuras anatómicas de pacientes atendidos endodónticamente, el año 2016, en la clínica Nexodent de la ciudad de Guayaquil, mediante el uso de sus tomografías previas a su tratamiento.

Materiales y métodos: Se analizaron 70 tomografías de 41 pacientes atendidos en el año 2016 en la clínica Nexodent de la ciudad de Guayaquil. Al momento de analizar cada tomografía se tomaron tres fotografías de cortes tomograficos: coronal, axial y sagital para obtener una información variada de su anatomía.

Resultados: De las tomografías revisadas, el $71 \%$ fue de género femenino. El 29\% de género masculino. Los resultados encontrados del número de conductos en las piezas dentales registradas señalan que el $56 \%$ de los casos presenta 1 sólo conducto. En cuanto a la variación anatómica de las piezas dentales estudiadas, se utilizó la clasificación de Vertucci. El $56 \%$ de las piezas dentales es de Tipo I, el $26 \%$ es de Tipo IV, el $11 \%$ es de Tipo II, y el 
restante son de Tipo V. Se analizó la distancia entre cada premolar maxilar hasta el seno maxilar y en promedio la distancia fue de 5,3 $\mathrm{mm}$. La distancia promedio de los premolares mandibulares hasta el foramen mentoniano fue de $6,21 \mathrm{~mm}$. La principal localización encontrada para el orificio del foramen apical fue el centro con el $58 \%$ de los casos.

Discusión: Se obtuvo mayoría de aciertos sobre los estudios realizados con los estudios de las referencias bibliográficas excepto en; La incidencia de los conductos en los segundos premolares superiores en que se obtuvo mayoria de un conducto en lugar de dos. En la distancia promedio del apice de los primeros premolares mandibulares con el agujero mentoniano en donde las distancias promedios fueron mayores. En la localizacion del foramen apical en la pieza \#35, en que hubo mayor localizacion del foramen en el centro y no hacia distal.

Conclusión: Se puede concluir que el mejor examen complementario para analizar la anatomía de conductos es la tomografía y que los resultados obtenidos en esta investigación no fueron muy distintos en comparación a investigaciones realizadas por otros autores.

Palabras clave: Anatomía radicular, premolares, número de conductos, seno maxilar, agujero mentoniano, foramen apical.

\section{Abstract}

Introduction: Endodontic treatment currently represents a large branch in the area of dentistry of importance for the preservation of dental pieces, which would be affected by the attack of external agents and compromise its functionality and aesthetics, but the complexity of the root Canals anatomy of all teeth in general still remains a problem, even more so in the premolars as these unlike other teeth, have different forms and quantity of root Canals. ${ }^{6}$

Purpose: The purpose of this study is to demonstrate the importance of knowledge about the root canal anatomy and its anatomical variations, in order to minimize the failure in an endodontic therapy.

Objective: The direct objective of this study is to determine the anatomical variations of upper and lower premolars and their relationship with anatomical structures of endodontically treated patients, in 2016, at the Nexodent Clinic of the city of Guayaquil, using their tomography prior to its treatment. Materials and methods: We analyzed 70 CT scans of 41 patients seen in 2016 at the Nexodent clinic in the city of Guayaquil.

At the moment of analyzing each tomography three photographs were taken: coronal, axial and sagittal to obtain al the information of its anatomy. Results: Of the CT scans reviewed, $71 \%$ were female, 29\% male. The results found of the number of root canals in the registered dental pieces indicate that $56 \%$ of the cases present 1 only conduit. Regarding the anatomical variation of the studied dental pieces, the Vertucci classification was used $56 \%$ of the teeth are Type I, 26\% are Type IV, 11\% are Type II, and the rest are Type $\mathrm{V}$. The average distance between the maxillary premolars to the maxillary sinus was $5.3 \mathrm{~mm}$. The mean distance from the mandibular premolars to the mental foramen was $6.21 \mathrm{~mm}$. The main location found for the apical foramen was the center with $58 \%$ of the cases.

Discussion: the mayority of the studies carried out with the studies of the bibliographical references where equal except in; The incidence of root canals in the upper second premolars where the mayority of one root was obtained instead of two. In the average distance of the apex of the first mandibular premolars with the mental foramen where the average distances were greater. In the location of the apical foramen in \# 35, in which there was greater location of foramen in the center and not distal.

Conclusion: It can be concluded that the best complementary exam to analyze the anatomy of root Canals is the tomography and that the results obtained in this investigation were not very different 
in comparison to investigations realized by other authors.

Key words: Root anatomy, premolars, maxillary sinus, mental foramen, apical foramen.

\section{Introducción}

El tratamiento endodontico representa en la actualidad una gran rama en el área de la odontología de importancia para la preservación de piezas dentales, que se verían afectadas por el ataque de agentes externos y que comprometen su funcionabilidad y estética. Un aspecto fundamental para realizar correctamente un tratamiento endodontico es el conocimiento de la configuración y forma de los conductos radiculares ${ }^{6}$ y la preparación químico-mecánica del sistema de conductos radiculares que tendrá cada pieza dentaria, pero la complejidad de la anatomía de los conductos de todos los dientes en general aún sigue siendo un problema, más aun en los premolares, ya que estos a diferencia de las demás piezas dentarias, tienen diferentes formas y cantidad de conductos radiculares. Estas diferencias pueden atribuirse a la presencia de importantes variaciones morfológicas apicales y a características propias de los diferentes grupos étnicos. ${ }^{1}$

La examinación radiográfica es un componente esencial en el manejo endodontico ${ }^{6-5}$, esto nos da aspectos de diagnostico, planeación del tratamiento, control y evaluación de los resultados. La radiografía nos proporciona información útil para conocer la anatomía de conductos radiculares y la proximidad de sus estructuras anatómicas adyacentes pero su precisión sobre la morfología del sistema de conductos radiculares es reducida porque proporciona una imagen bidimensional de una estructura tridimensional. ${ }^{6-5}$

La CBCT recoge datos de volumen por medio de una rotación simple con un haz de rayos $\mathrm{x}$ en forma de cono, y detectores de dos dimensiones, y proporciona imágenes de alta calidad diagnostica, con tiempos cortos de exposición y bajas dosis de radiación. ${ }^{(5)}$ Las variaciones de la anatomía del sistema de conductos radiculares han sido clasificadas por varios autores. Weine y col, categorizó el sistema de conductos radiculares en cuatro tipos básicos. En otro estudio, Vertucci identificó ocho configuraciones del espacio de los conductos. Posteriormente, Sert \& Bayirli complementaron la clasificación de Vertucci. ${ }^{5}$

El propósito de este estudio es demostrar la importancia del conocimiento sobre la anatomía radiculary sus variaciones anatómicas, para minimizar el fracaso al realizar una terapia endodontica. El objetivo directo de este estudio es determinar las variaciones anatómicas de premolares superiores e inferiores y su relación con estructuras anatómicas de pacientes atendidos endodonticamente, el año 2016, en la clínica Nexodent de la ciudad de Guayaquil, mediante el uso de sus tomografías previas a su tratamiento.

\section{Materiales y Metodos}

Este fue un estudio observacional, analítico y descriptivo, transversal.

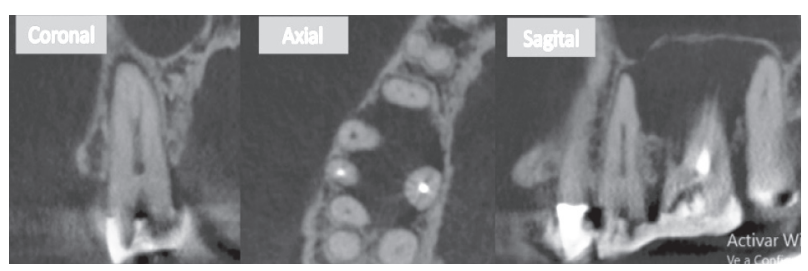

Se analizaron 70 tomografías de 41 pacientes atendidos en el año 2016 en la clínica Nexodent de la ciudad de Guayaquil, donde se realizan tratamientos endodonticos en premolares. Se realizo el permiso especifico de la clínica Nexodent para obtener sus archivos. Al momento de analizar cada tomografía se tomaron tres fotografías de cortes tomograficos: coronal, axial y sagital a las tomografías de los premolares en estudio para poder obtener una información variada de su anatomía.

Posteriormente, se midió la distancia del ápice de los premolares superiores hacia el seno maxilar y la distancia de los ápices de los premolares inferiores hacia el agujero mentoniano.

Con la información obtenida se tabularon los datos y finalmente se analizaron los resultados con la 
finalidad de determinar la incidencia del número de conductos, la incidencia de la clasificación anatómica según vertucci, que la proximidad de los premolares superiores con el seno maxilar, la proximidad de los premolares inferiores con el agujero mentoniano y la localización de los ápices en los premolares superiores e inferiores.

\section{Resultados}

De las tomografías revisadas, el $71 \%$ fue de género femenino. El 29\% de género masculino.

Con respecto al número de conductos encontrados no se puede afirmar que el género influye ya que la diferencia entre hombres y mujeres no es muy alta, y más si consideramos que solo el $29 \%$ de las tomografías fueron del sexo masculino. En el género femenino, el 58\% tuvieron 1 conducto y en el género masculino fueron el $50 \%$.

Los resultados encontrados del número de conductos en las piezas dentales registradas señalan que el $56 \%$ de los casos presenta 1 sólo conducto. No se encontraron casos en donde hayan más de 2 conductos.

Con respecto a si son Premolares Superiores o Maxilares, la mayoría presentan 2 conductos (56\%), en tanto que en los Premolares Inferiores la mayoría presenta 1 conducto (76\%).

Entre los primeros y segundos premolares, se encontró que para los primeros el $71 \%$ tuvieron 2 conductos, mientras que en los segundos, la mayoría tuvieron sólo 1 conducto ( $81 \%$ de los casos)

En la pieza \#14 y \#24, correspondientes a primer premolar maxilar se encontró una mayor presencia de 2 conductos (90\% y $85 \%$ respectivamente). Mientras que, en la pieza \#15 el 63\% tuvo 1 conducto y en la pieza \#25 el $86 \%$ se encontró 1 conducto.

Para los premolares inferiores o mandibulares, en la pieza \#34 el 75\% de los casos presentó 1 conducto y el $25 \%$ restante se encontró 2 conductos. En las piezas \#35 y \#44 en la mayoría presentó sólo 1 conducto, siendo el $60 \%$ y $57 \%$ respectivamente. En cuanto a la pieza \#45 sólo se encontraron casos de 1 conducto.

En cuanto a la variación anatómica de las piezas dentales estudiadas, se utilizó la clasificación de Vertucci. Según esta clasificación, no fueron encontrados casos en Tipo III, Tipo VI, Tipo VII y Tipo VIII. El 56\% de las piezas dentales es de Tipo I (esto corresponde a la misma cantidad de casos que tienen 1 conducto), el 26\% es de Tipo IV, el $11 \%$ es de Tipo II, y el restante son de Tipo V.

En los premolares superiores, el $44 \%$ de las piezas dentales son de Tipo I, seguidas por el Tipo IV, $36 \%$; Tipo II, $16 \%$ y Tipo V el $4 \%$. En los Premolares Inferiores, el $76 \%$ son de Tipo I, el 12\% Tipo V, el $8 \%$ Tipo IV y el $4 \%$ de Tipo II.

En la pieza \#14, se encontraron más casos de Tipo IV (60\%), seguidos por el Tipo II (30\%), y el restante siendo de Tipo I. Para la pieza \#15, el 63\% son de Tipo I, el 25\% de Tipo IV y el 13\% de Tipo V. En la pieza \#24, la mayoría son del Tipo I (46\%), el 31\% son de Tipo II, el $15 \%$ de Tipo I, y el restante $8 \%$ son de Tipo V.

En cuanto a la pieza \#34, sólo se encontraron casos de Tipo I y Tipo 5, siendo el 75\% de Tipo I. Para la pieza \#35, el 60\% de los casos fueron de Tipo l, y los restantes entre Tipo IV y V (20\% cada uno). En la pieza \#44, la mayoría también fue de Tipo I con el 57\%, mientras que en los tipos II, IV y V se encontró el 14\% de los casos. En la pieza \#45 consecuentemente con que sólo se encontró 1 conducto, todas son de Tipo I. Se analizó la distancia entre cada premolar maxilar (\#14, \#15, \#24, \#25) hasta el seno maxilar y en promedio la distancia fue de $5,3 \mathrm{~mm}$.

Para los primeros premolares superiores (\#14 y \#24) la distancia fue mayor que la encontrada respecto a los segundos premolares superiores.

La distancia hasta el seno maxilar para la pieza dental \#14 fue en promedio de 7,8 mm, para la pieza dental \#24 de 6,4 mm, para la \#25 de 4,2 mm, y la menor fue para la pieza dental \#15, con $2,3 \mathrm{~mm}$ en promedio hasta el seno maxilar. 
Estas distancias también fueron clasificadas en 3 categorías en función de los valores que tomaba, siendo "Cerca" cuando la distancia era menor a 3mm, "Moderada" para distancias entre 3 y 4 mm, y "Lejos" cuando la distancia es mayor a $4 \mathrm{~mm}$.

En los registros analizados se nota que la mayoría de los premolares superiores se encuentran "Lejos" del piso del seno maxilar (58\%) y "Cerca" el 36\%.

Para la pieza \#14, el $80 \%$ de los premolares se categorizaron al respecto de la distancia al piso del seno maxilar como "Lejos", y el 20\% restante dividido entre las otras dos categorías. En la pieza \#15, el 75\% tenía distancias menores a 3mm (Cerca), el 13\% con distancias "Moderada" y el otro 13\% "Lejos". En la pieza \#24, la mayoría tuvo distancias $>4 \mathrm{~mm}$ (69\%) y un $23 \%$ fueron $<3 \mathrm{~mm}$. En cambio para la pieza \#25, no hubieron casos de distancias "Moderadas", el 57\% fueron "Lejos" y el 43\% restante "cerca".

La distancia promedio de los premolares mandibulares hasta el foramen mentoniano fue de $6,21 \mathrm{~mm}$.

Entre los primeros premolares mandibulares las distancias promedios fueron mayores, teniendo 7,28 $\mathrm{mm}$ para las piezas dentales \#34 y de $8,47 \mathrm{~mm}$ para las piezas dentales \#44.

En tanto que para los segundos premolares mandibulares \#35 y \#45, las distancias fueron de 5,33 y $4,47 \mathrm{~mm}$ en promedio.

Estas distancias también fueron clasificadas en categorías, siendo Cerca en los casos en que la distancia hasta el conducto mentoniano fue menor a $4 \mathrm{~mm}$, Moderado cuando el valor se encontró entre 4 y $8 \mathrm{~mm}$ y Lejos cuando la distancia registrada superaba los $8 \mathrm{~mm}$.

En general, la mayoría de las piezas dentales mandibulares, tuvieron distancias "Moderadas" hasta el conducto mentoniano, siendo del 52\%, y con distancias "Cerca" y "Lejos" hubieron igual porcentaje de casos ( $24 \%$ cada una).

Para los primeros premolares mandibulares no se registraron casos con distancias menores a $4 \mathrm{~mm}$ ("Cerca"). Para la pieza \#34, el 75\% de las distancias fueron "Moderadas", y el restante 25\% fueron "Lejanas". Para la pieza \#44, la mayoría también tuvieron distancias "moderadas", 57\%. En tanto para los segundos premolares mandibulares no hay mucha similitud entre ellos, notando que para el \#35 la mayoría de las distancias fueron categorizadas como "Moderadas" (60\%) y para el \#45, la mayoría de las distancias fue "Cerca" con el 56\%.

Para analizar la localización del foramen apical entre los premolares observados, se clasificará inicialmente entre los premolares que terminaron en 1 ápice (Tipo I y II de la clasificación de Vertucci), y aquellos que terminaron en 2 ápices (Tipos IV y V de la clasificación de Vertucci).

De los casos registrados, el 67\% terminaron en un ápice y el 33\% en 2 ápices. Para los premolares superiores el $60 \%$ terminaron en 1 ápice y el restante 40\% en 2 ápices, para los premolares inferiores la mayoría terminaron en 1 ápice y sólo un 20\% terminaron en 2 ápices.

\section{Con un ápice}

Entre las piezas dentales con 1 ápice, el 40\% tienen una localización "central", seguidos por la localización "Distal" con el 38\%, en menores porcentajes se encontraron con localizaciones Mesial , Bucal y Palatino.

Para los premolares maxilares, el orificio del foramen apical se ubicó en Centro y en Distal el 33\% en cada uno, y en Bucal, Lingual y Mesial un $11 \%$ en cada uno. Para los premolares mandibulares, la mitad de ellos tiene el orificio del foramen apical en el "Centro", sólo el $5 \%$ está localizado en Mesial, el $45 \%$ restante se encuentra en Distal.

Para la pieza dental \#14, el $75 \%$ de los casos se localizan en Centro, y el 25\% en Distal. Para la pieza \#24, hay igual porcentaje entre las localizaciones Centro, Distal y Mesial (33\% cada una). Para la pieza dental \#15, el 80\% está localizada en Distal y el restante en Lingual. En cambio para la pieza \#25, la 
mayor parte se ubica en Centro (33\%), seguida por Bucal (25\%), sólo un $8 \%$ se ubica en Mesial.

En los premolares mandibulares no existen casos con localizaciones Bucal y Lingual. Los primeros premolares mandibulares \#34 y \#44, la mayoría tienen el orificio del foramen ubicado en Centro (67\% y 60\% respectivamente) y lo restante en Distal. Para la pieza dental \#35, están ubicados en Centro, Distal y Mesial, el 33\% en cada uno; mientras que en la \#45, el 56\% está en Distal y el restante en Centro.

\section{Con dos ápices}

Entre los premolares en los que se encontraron 2 ápices, la localización se dividió entre Vestibular y Palatino.

Para los ápices vestibular, la principal localización del orificio del foramen es Centro, con el 65\%, seguida por Bucal con el 26\%, en Mesial sólo hubo el 9\%.

Para los premolares superiores el 67\% se localizaron en Centro y el restante en Bucal. En los premolares mandibulares el $60 \%$ se ubicó en Centro y el $40 \%$ en Mesial.

En las piezas dentales superiores \#14 y \#15, el 67\% se encontraron en Centro y el 33\% restante en Bucal. En la pieza \#24, también la mayoría se ubicó en el centro (71\%), seguida por Bucal. Para el premolar \#25, estuvieron ubicados en igual proporción entre las localizaciones Centro y Bucal. Ninguno tuvo localización del orificio del foramen en Mesial. En las piezas dentales inferiores \#35 y \#44 los casos estuvieron divididos entre Centro y Mesial (50\% cada una), y en la pieza \#34 todos los casos estuvieron ubicados en Centro. No se regstraron casos de la pieza dental \#45 con 2 ápices.

Para los ápices palatino, como en Vestibular, la principal localización del orificio del foramen es Centro, pero con un porcentaje mayor siendo del $87 \%$, seguida por Lingual con el $9 \%$, y por Distal con el $4 \%$.

Tanto para los premolares maxilares como mandibulares, la mayoría de los ápices están en
Centro. En los superiores el $11 \%$ está en Lingual o palatino y en los inferiores el $20 \%$ está en Distal.

Para las piezas dentales superiores como ya se observó en el gráfico anterior los mayores porcentajes se ubican en Centro. En los primeros premolares superiores, el ápice palatino tuvo como segunda ubicación Lingual, siendo del 17\% para la pieza \#14 y del 14\% para la pieza \#24. En cuanto que para los segundos premolares superiores, todos los casos del ápice palatino se ubicaron en Centro.

En los premolares inferiores \#34 y \#35, el 100\% de los casos se localizaron en Centro, mientras que en la pieza \#44 se encontraron casos en Centro y Distal (50\% cada uno). No se regstraron casos de la pieza dental \#45 con 2 ápices.

En general para todos los casos, con 1 y 2 ápices, la principal localización encontrada para el orificio del foramen apical fue el Centro con el 58\% de los casos, mientras que la localización que menos se encontró fue Lingual o Palatino.

En las piezas dentales mandibulares, más de la mitad se encontraron con localización Centro (59\%), seguida por Bucal y Distal con el 14\% cada uno. En los premolares inferiores también se muestra que la principal localización es el centro con el 57\%, seguida de Distal con el 33\% y el 10\% restante ubicado en Mesial.

En la pieza dental \#14, el 75\% de los orificios se localizaron en Centro, seguido por el 13\% en Bucal. En la pieza dental \#15 el 45\% se ubicó en Centro y el $36 \%$ en Distal. En estas 2 piezas no hubieron casos de localizaciones en Mesial. Para las piezas dentales \#24 y \#25, la mayoría también se ubicaron en Centro, con el $65 \%$ y $44 \%$ respectivamente. En la pieza dental \#24, se localizaron en Bucal, Distal y Mesial el 10\% de los casos en cada uno, y sólo el 5\% en Palatino. Para la \#25, tuvo un25\% de casos ubicados en Bucal, y en Distal y Lingual $13 \%$ cada uno.

En las piezas dentales mandibulares no existen casos con localización Bucal y Lingual. Para las piezas \#34, 
\#35, \#44 la mayoría de los casos tuvieron orificios ubicados en el Centro. Para la pieza \#34, el 80\% se ubicó en Centro y el 20\% en Distal. Para la pieza \#35, el $57 \%$ fue en Centro, seguido del $29 \%$ en Mesial. En la \#44, el 56\% se ubicó en Centro, el 33\% en Distal y el $11 \%$ en Mesial. Para la pieza \#45, en cambio, fue mayor el porcentaje en Distal (56\%) que en Centro (44\%).

\section{Discusión}

Greco, Machado et al. En España realizaron un estudio en donde analizaron todos los premolares, tanto superiores como inferiores y primeros como segundos y obtuvieron este resultado que nos demuestra su gran variabilidad.

Los primeros premolares superiores con un solo conducto tienen un porcentaje de $5.88 \%$, de dos conductos $88,22 \%$ y de tres conductos $5,88 \%$.

Los segundos premolares superiores con un solo conducto $39,65 \%$, de dos conductos $60,31 \%$ y de tres $0 \%$. En los primeros premolares inferiores, con un solo conductos $68,28 \%$ y de dos conductos $31,8 \%$.

En los segundos premolares inferiores con un solo conducto es de $73,91 \%$ con dos conductos de 26,08\%.' Acertando con este presente estudio en que se demostró que en los primeros premolares superiores se encontró una mayor presencia de 2 conductos ( $90 \%$ en la pieza \# 14 y $85 \%$ en pieza \#24 respectivamente). A diferencia del resultado obtenido para la incidencia de los conductos en los segundos premolares superiores en la pieza \#15 el $63 \%$ tuvo 1 conducto y en la pieza \#25 el $86 \%$ se encontró 1 conducto.

En el estudio de los primeros premolares mandibulares se acerto con el estudio presente en que la pieza \#34 el 75\% de los casos presentó 1 conducto y el $25 \%$ restante se encontró 2 conductos, y en la pieza \#44 presentó sólo 1 conducto con el $57 \%$ de los casos y tambien se acerto en cuanto a los segundos premolares inferiores en las piezas \#35 se presentó sólo 1 conducto con el $60 \%$ y en la pieza \#45 sólo se encontraron casos de 1 conducto.
Celikten, Orhan et al. Realizaron un estudio sobre la configuración de la anatomía radicular mediante la clasificación de vertucci y su estudio presento que los premolares maxilares tanto, primeros como segundos, eran tipo IV (76,8\%) y tipo I (49,4\%) asi también se demostró que la configuración anatómica en los premolares mandibulares es de tipo (93\%).(2) Acertando con este estudio en que los tipos IV y I son los mas encontrados en los premolares superiores, el 44\% de las piezas dentales son de Tipo I, seguidas por el Tipo IV, 36\%; Tipo II, 16\% y Tipo V el 4\%. Y que en los Premolares Inferiores, el $76 \%$ son de Tipo I, el 12\% Tipo V, el $8 \%$ Tipo IV y el 4\% de Tipo II.

Kilic et al, realizaron un estudio tomografico para medir la distancia de los ápices de los premolares maxilares hacia el piso del seno maxilar y dio como resultado:

Un distancia de $8.42 \mathrm{~mm}$ para los primeros premolares \#14 y 6.58 para los primeros premolares \#24. Para los segundos premolares premolares \#15 $3.75 \mathrm{~mm}$ y $3.73 \mathrm{~mm}$ para los segundos premolares \#25. ${ }^{3}$ Acertando relativamente con el estudio presente en que la distancia hasta el seno maxilar para la pieza dental \#14 fue en promedio de 7,8 mm, para la pieza dental \#24 de 6,4 mm, para la \#25 de 4,2 mm, y la menor fue para la pieza dental \#15, con 2,3 mm en promedio hasta el seno maxilar.

Villalobos y Muñoz realizaron un estudio sobre la distancia entre el foramen mentoniano con el ápice los premolares mandibulares, lo que dio como resultado que la distancia promedio del ápice del primer premolar mandibular al agujero mentoniano es de 4,38mm y del ápice del segundo premolar mandibular hacia el agujero mentoniano es de $4,75 \mathrm{~mm}^{4}$ No concordando con el estudio realizado en que se encontró que entre los primeros premolares mandibulares las distancias promedios fueron mayores, teniendo $7,28 \mathrm{~mm}$ para las piezas dentales \#34 y de $8,47 \mathrm{~mm}$ para las piezas dentales \#44. En tanto que para los segundos premolares mandibulares si se acerto relativamente con el presente estudio en que los segundos premolares \#35 y \#45, las distancias fueron de 5,33 y 4,47 mm en promedio. 
Cardona y Fernández, investigaron toda la anatomía de los dientes en los cuales podemos definir según su estudio las localizaciones de los ápices que dieron que en el primer premolar maxilar, si tiene una sola raíz, el alveolo está cerca en relación con la placa ósea vestibular y si tiene dos raíces, la raíz vestibular está alineada adyacente a la placa cortical vestibular, mientras la raíz palatina estará centralmente localizada, en el segundo premolar maxilar es común que posea un solo conducto y su ápice seria desviado hacia distal. En los primeros premolares mandibulares el ápice se desvía hacia distal en la mayoría de veces, y en los segundos premolares mandibulares el foramen apical se desvía hacia distal. ${ }^{5}$ Acertando relativamente con el estudio realizado en que se vio que en la pieza dental \#14, el 75\% de los orificios se localizaron en Centro, seguido por el 13\% en Bucal y para las piezas dentales \#24, la mayoría también se ubicaron en Centro, con el $65 \%$ y se localizaron en Bucal, Distal y Mesial el 10\% de los casos, y sólo el 5\% en Palatino. En los segundos premolares maxilares la pieza dental \#15 el 45\% se ubicó en Centro y el 36\% en Distal.Para la \#25, tuvo un $25 \%$ de casos ubicados en Bucal, y en Distal y Lingual 13\%.

En las piezas dentales mandibulares tambien se acerto con el estudio rrealizado, en que se obtuvo que para la pieza \#34, el $80 \%$ se ubicó en Centro y el $20 \%$ en Distal y en la \#44, el 56\% se ubicó en Centro, el $33 \%$ en Distal y el $11 \%$ en Mesial y para la pieza \#45, fue mayor el porcentaje en Distal (56\%) que en Centro (44\%), solo no hubo concordancia en la pieza \#35, en que el 57\% fue en Centro, seguido del 29\% en Mesial.

\section{Conclusión}

Se puede concluir que el mejor examen complementario para analizar la anatomía de conductos es la tomografía y que los resultados obtenidos en esta investigación no fueron muy distintos en comparación a investigaciones realizadas de otros autores, que la incidencia del numero de conductos varia según cada pieza dentaria con una mayoría de 1 conducto en los premolares inferiores y dos conductos en los superiores, en cuanto al tipo de conductos prevalecen el tipo I y tipo IV en premolares superiores y tipo I en premolares inferiores. En la distancia de los ápices de los premolares superiores al seno maxilar dio un promedio de 5,3 mm. En la distancia de los apices de los premolares inferiores hacia el agujero mentoniano de un promedio de 6,21 $\mathrm{mm}$. En cuanto a la localizacion encontrada para el orificio del foramen apical fue el centro con el $58 \%$ de los casos.

\section{Bibliografía}

1. Greco-Machado Y, García-Molina J, Lozano-De Luaces V, Manzaranes-Céspedes M. Morfología de los conductos radiculares de premolares superiores e inferiores. Endodoncia. 2009;27(1):13 - 18.

2. Celikten B, Orhan K, Aksoy U, Tufenkci P, Kalender A, Basmaci $F$ et al. Cone-beam CT evaluation of root canal morphology of maxillary and mandibular premolars in a Turkish Cypriot population. BDJ Open. 2016;2:15006.

3. Kilic C, Kamburoglu K, Pehlivan S, Yuksel P, Ozen T. An Assessment of the Relationship between the Maxillary Sinus Floor and the Maxillary Posterior Teeth Root Tips Using Dental Conebeam Computerized Tomography. European Journal of Dentistry. 2010;4:462 - 467

4. Villalobos Blanco A, Muñoz Solis A. Ubicación y distancia del foramen mentoniano con respecto a los ápices de las premolares inferiores. Endodoncia. 2014;:1 - 14.

5. Cardona-Castro JA, Fernandez-Grisales R. Anatomía radicular, una mirada desde la micro-cirugia endodontica: Revisión. Rev. CES Odont. 2015; 28(2): 70-99

6. Ozcan G, Bulut D, Kose E, Sekerci A, Canger E, Sisman Y. Evaluation of root morphology and root canal configuration of premolars in the Turkish individuals using cone beam computed tomography. European Journal of Dentistry. 2015;9(4):551.

7. Sousa T, Haiter-Neto F, Nascimento E, Peroni L, Freitas D, Hassan B. Diagnostic Accuracy of Periapical Radiography and Cone-beam Computed Tomography in Identifying Root Canal Configuration of Human Premolars. Journal of Endodontics. 2017;43(7):1176-1179.

8. Borges A, Gonçales Orçati D, Miranda P, Faitaroni F, Mamede Neto L, Ricci Volpato L. Estudio de las variaciones anatómicas de los premolares: reporte de cuatro casos clínicos. Acta Odontológica Venezolana. 2013;51(4).

9. Bürklein S, Heck R, Schäfer E. Evaluation of the Root Canal Anatomy of Maxillary and Mandibular Premolars in a Selected German Population Using Cone-beam Computed Tomographic Data. Journal of Endodontics. 2017;43(9):1448-1452.

10. Oporto V G, Fuentes F R, Soto P C. Variaciones Anatómicas Radiculares y Sistemas de Canales. International Journal of Morphology. 2010;28(3).

11. Habib A, Kalaji M, Al saysd T, Al jawfi K. Root canal configurations of the first and second mandibular premolars in the population of north Syria. Journal of Taibah University Medical Sciences. 2015;10(4):391-395.

12. Vertucci F, Gegauff A. Root canal morphology of the maxillary first premolar. The Journal of the American Dental Association. 1979;99(2):194-198. 
13. Arana-Fernández de Moya E, Buitrago-Vera P, Benet-Iranzo F, Tobarra-Pérez E. Tomografía computerizada: introducción a las aplicaciones dentales. RCOE. 2017;11(3):311 - 322.

14. Baroudi K, Kazkaz M, Sakka S, Tarakji B. Morphology of root canals in lower human premolars. Nigerian Medical Journal. 2012;53(4):206.

15. Vertucci F. Root canal morphology and its relationship to endodontic procedures. Endodontic Topics. 2005;10(1):3-29.

16. Espadas-Sánchez C, Alvarado-Cárdenas G, Vega-Lizama E, López-Villanueva M, Ramírez-Salomón M. Identificación de la morfología de primeros premolares maxilares mediante cortes transversales. Tame. 2013;2(5):138 - 142.

17. Jayasimha R, Mylswamy S. Root canal morphology of maxillary second premolars in an Indian population. Journal of Conservative Dentistry. 2010;13(3):148.

18. Abraham SB, Gopinath VK. Root canal anatomy of mandibular first premolars in an Emirati subpopulation: A laboratory study. Eur J Dent 2015;(9):476-82.

19. Arslan H, Capar I, Akcay M, Ertas E, Ertas H. A cone-beam computed tomographic study of root canal systems in mandibular premolars in a Turkish population: Theoretical model for determining orifice shape. European Journal of Dentistry. 2015;9(1):11.

20. von Arx T, Fodich I, Bornstein M. Proximity of Premolar Roots to Maxillary Sinus: A Radiographic Survey Using Cone-beam Computed Tomography. Journal of Endodontics. 2014;40(10):15411548.

21. Lavasani S, Tyler C, Roach S, McClanahan S, Ahmad M, Bowles W. Cone-beam Computed Tomography: Anatomic Analysis of Maxillary Posterior Teeth-Impact on Endodontic Microsurgery. Journal of Endodontics. 2016;42(6):890-895.
22. Singh S, Pawar M. Root Canal Morphology of South Asian Indian Mandibular Premolar Teeth. Journal of Endodontics. 2014;40(9):1338-1341.

23. Johnsen G, Dara S, Asjad S, Sunde P, Haugen H. Anatomic Comparison of Contralateral Premolars. Journal of Endodontics. 2017;43(6):956-963.

24. Ring J, Ring K. Rare Root Canal Configuration of Mandibular Second Premolar Using Cone-beam Computed Tomographic Scanning. Journal of Endodontics. 2017:1-4.

25. Khedmat S, Assadian H, Saravani A. Root Canal Morphology of the Mandibular First Premolars in an Iranian Population Using Cross-sections and Radiography. Journal of Endodontics. 2010;36(2):214-217.

26. Abella F, Teixidó L, Patel S, Sosa F, Duran-Sindreu F, Roig M. Cone-beam Computed Tomography Analysis of the Root Canal Morphology of Maxillary First and Second Premolars in a Spanish Population. Journal of Endodontics. 2015;41(8):1241-1247.

27. Peña Angulo M, Gutiérrez Báez R, Rujano Carrillo J, Orellana Jaimes N, Marín Altuve E. Estudio anatómico de los conductos radiculares del primer premolar superior con dos raíces mediante la técnica de diafanización dental. Revista odontologica de los andes. 2014;9(1):16 - 22.

28. Sulaiman A, Dosumu O, Amedari M. MAXILLARY FIRST PREMOLAR WITH THREE ROOT CANALS: A CASE REPORT. Annals of Ibadan Postgraduate Medicine. 2013;11(2):105 -108.

29. Relvas J, de Carvalho F, Marques A, Sponchiado E, Garcia L. Endodontic Treatment of Maxillary Premolar with Three Root Canals Using Optical Microscope and NiTi Rotatory Files System. Case Reports in Dentistry. 2013;2013:1-4.

30. 20. Kartal N, Özçelik B, Cimilli H. Root canal morphology of maxillary premolars. Journal of Endodontics. 1998;24(6):417-419. 